\title{
Genetic Evidence for Hybridization in an Interspecific Cross in the Genus Sordaria
}

\author{
By L. A. LEWIS* \\ Department of Biological Sciences, Columbia University, \\ New York, New York 10027, U.S.A.
}

(Accepted for publication 15 September 1969)

\begin{abstract}
SUMMARY
When the two heterothallic species Sordaria heterothallis and S. thermophila are crossed, two types of viable ascospores are recovered. The first, the mature black ascospore, is produced with a mature eight-spored ascus formed from an intraspecific fusion of nuclei of the same mating type. The second, the non-pigmented germinable ascospore, is produced by the interspecific fusion of nuclei of opposite mating types within an ascus in which all other ascospores are aborted. The non-pigmented ascospore is the product of the mixed genomes of the two species. It is a recombinant spore from which either species may be obtained by the partial substitution of its genome through successive backcrosses. Its inability to develop pigmentation, the irregular growth patterns of some of the strains which it produces and their inability to react in complementation tests, appear to be the result of a lack of affinity between the two species.
\end{abstract}

\section{INTRODUCTION}

Two new heterothallic species, Sordaria heterothallis (Fields \& Maniotis, 1963) and $S$. thermophila (Fields, 1968), have recently been described. Previously reported studies on the hybridization experiments between these species (Fields, 1963; Lewis, I968a, 1969) have indicated that the primary result was the formation of perithecia in which most of the asci were aborted at varying stages of maturation, and in which an occasional mature eight-spored ascus was recovered. These asci (recovered at a frequency of I per I0,000 asci examined) were found to have been homozygous for the mating type factor. Lewis (I968 $b$ and 1969) described evidence which suggests that they were formed by the intraspecific fusion of nuclei of the same mating type. Although most of the spores formed in these asci were normal in colour and shape and showed reasonable viability, these studies also indicated that aneuploid ascospores were formed during meiosis and that the resultant loss of chromosomes contributed to the inviability observed in some ascospores.

Another type of viable ascospore produced from the hybridization attempts was the non-pigmented germinable ascospore. The aim of this study was to determine whether this ascospore was the product of intra- or interspecific nuclear fusions. Proof of the latter would determine whether hybridization had occurred between the two species.

* Present address: Dept. of Biological Sciences, York College of the City University of New York, Flushing, New York 11365 , U.S.A. 


\section{MATERIALS}

Wild-type strains of Sordaria heterothallis and $S$. thermophila were supplied by Dr W. G. Fields. The wild-type strains of $S$. heterothallis were designated as WT 5- $A$ and WT $16-a$, and those of $S$. thermophila as WT 7- $A$ and WT $17-a$. In each case $A$ and $a$ refer to the two mating types. Differences between the two species, and descriptions of the media, the cultural conditions employed and the techniques used in the isolation, characterization and mapping of mutants have been previously reported (Lewis, 1969). The $S$. heterothallis mutants used in this study were $s t u_{1}, s t u_{4}, s t u_{5}, s t u_{6}$ (strains with stunted growth) and $o l_{3}$ (olive-green ascospores). Those of $S$. thermophila were col $_{1}$, $\mathrm{Col}_{2}, \mathrm{Col}_{3}$ (strains with a colonial type of growth) and $t$ (tan coloured ascospores). In $S$. heterothallis, $s t u_{1}$ and $s t u_{6}$ were $0 \cdot I$ units apart and $\mathrm{I} \cdot 0$ unit from the mating type locus. $S t u_{4}$ and $s t u_{5}$ were $2 \cdot 0$ units apart on another chromosome.

Several types of interspecific crosses were made: (I) Between the two wild-type strains. (2) $S t u_{6} \times t$ (cross no. 4, Table I). (3) Heterocaryons forced between $s t u$ strains of Sordaria heterothallis were crossed with wild-type strains of $S$. thermophila (crosses I to 3, Table I). From these crosses it was hoped to determine whether the $f_{1}$ progeny (I) segregated only as parentals and whether strains recombinant for the markers in the constituent strains of the heterocaryon could be recovered; (2) were the products of interspecific fusions. Progeny were analysed for recombination between the stu markers and the mating type factors in order to determine whether the non-pigmented germinable ascospores were derived from interspecific nuclear fusions. $f_{1}$ strains produced by non-pigmented germinable ascospores were classified to species by backcrossing to wild-type strains of both species. The genotypes of stunted progeny from interspecific crosses were determined by complementation tests with $s t u_{1,4,5}$ and $s t u_{6}$ of $S$. heterothallis and with the restricted colonial mutants of $S$. thermophila.

\section{RESULTS}

Fields (1963) carried out the initial interspecific matings between Sordaria heterothallis and $S$. thermophila. He found that there was an abundant production of perithecia in which almost all the asci contained aborted protoplasts or delimited ascospores which did not mature. He also observed (personal communication) that these 'immature' ascospores occasionally germinated.

A closer study of these non-pigmented germinable ascospores showed that they were produced in asci which contain no black spores, and in which only one spore germinated. The other seven ascospores in the asci showed various degrees of abortion and no germination (Pl. I, fig. I). These asci were produced at the rate of 20 to 30 per perithecium, and the delimited ascospores ranged in colour from hyaline to beige. Most asci contained eight aborted ascospores and made up the bulk of the cluster in interspecific matings (Pl. I, fig. 2). The germinable ascospores germinated without any of the pre-germination activation that was mandatory for Sordaria heterothallis and $S$. thermophila. The ascospores of $S$. heterothallis are only known to germinate when incubated on a medium containing $7 \%$ sodium acetate (Fields \& Maniotis, I963), and those of $S$. thermophila require a $2-\mathrm{hr}$ heat shock treatment and incubation on a medium containing $0.55 \%$ ammonium acetate (Lewis, 1969).

Non-pigmented germinable spores isolated from wild-type interspecific matings 


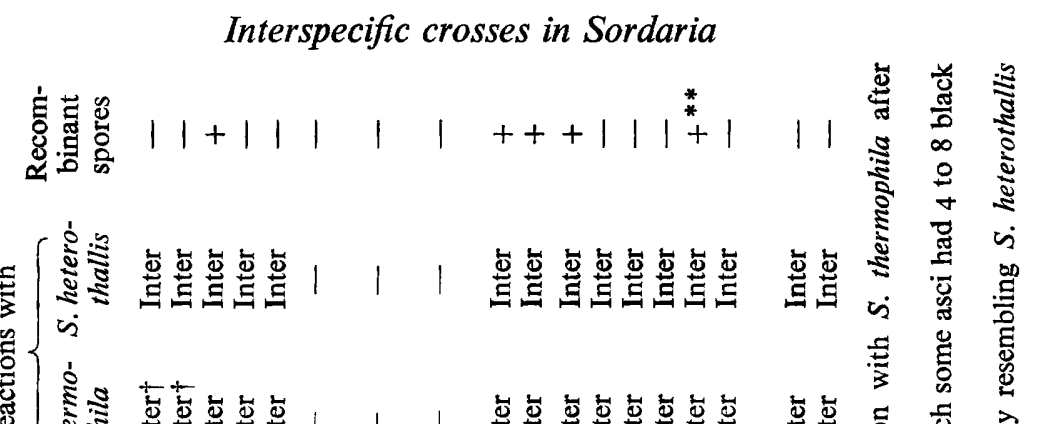

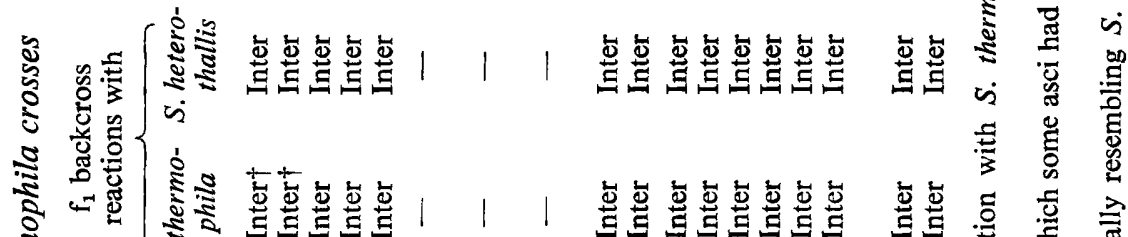

$36 \mathbf{I}$

ฐ

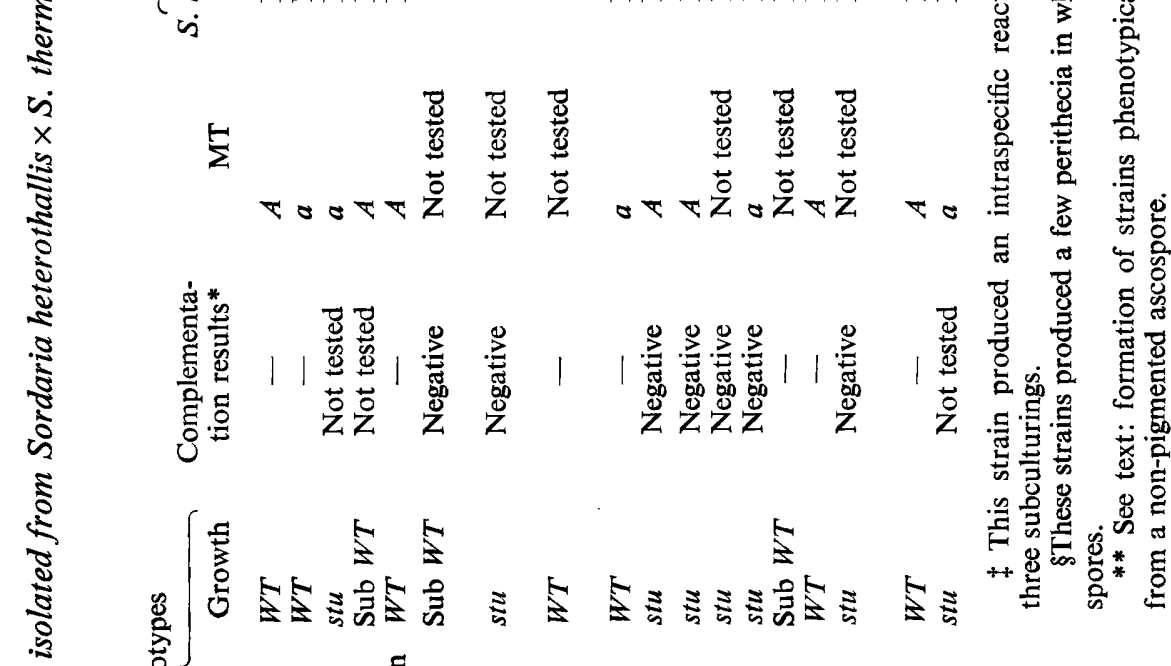

ปั

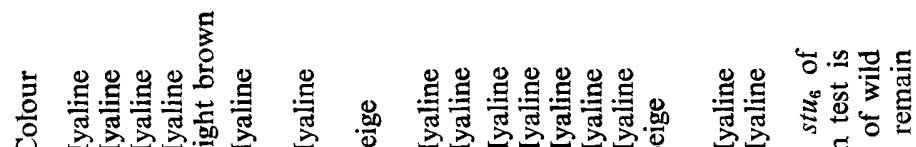

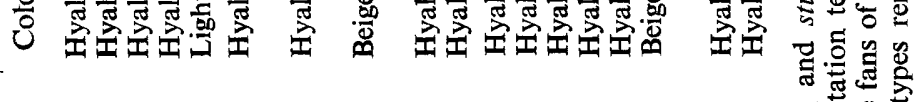

章

ב⿱艹

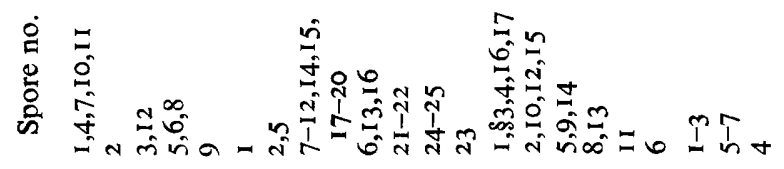

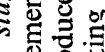

소을 염

की

政

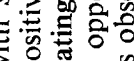

员苟药

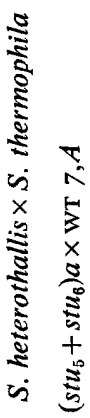

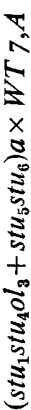

艺《溇

额管㤩

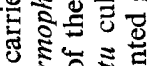

논

的总递

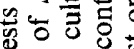

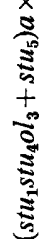

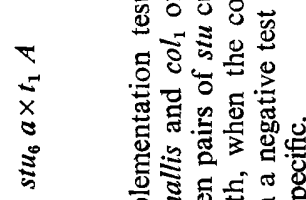

岕这 -

N

- 45 
showed two basic types of growth, normal wild type growth and a sub-wild type growth. The sub-wild type growth microscopically had an abnormal mycelial development, with much spiralling and more branching than the wild type. The growth rate was slower than the wild type, but the colony eventually grew to the edge of the Petri dish. In some sub-wild type strains, the colony gave rise to numerous sectors. These observations and the inability of the spores to develop normal pigmentation suggest that factors inhibiting the phenotypic expression of wild type were present in these non-pigmented ascospores, although some had become sufficiently mature physiologically to germinate and grow. When strains from the non-pigmented germinable ascospores were crossed among themselves, the asci produced showed an even greater degree of ascospore abortion than that produced in interspecific crosses. The nonpigmented germinable ascospores produced showed no phenotypic differences from their $f_{1}$ counterparts, and their mycelial strains had similar characteristics.

Progeny from the four matings shown in Table I are described phenotypically. $f_{1}$ cultures showed the phenotypes of either the mutant parental strains used in these matings, the sub-wild type mycelium, with its characteristic irregular growth and sectoring, or wild type growth with the normal phenotype. Thirty-five slow growing isolates were tested for complementation with stunted and colonial strains of both species. The results in Table I show that no complementation took place. It was assumed that failure of complementation might be due to the recombinant nature of the non-pigmented ascospores.

Twenty-nine of the isolated non-pigmented germinable ascospores were backcrossed to the wild type strains of both species. All strains showed an interspecific reaction with both species. This result led to the conclusion that the genomes of these strains were interspecifically recombinant, and that both parents contributed to their genotypes. Genetic proof of this is described in the following sections.

Twelve of the $f_{1}$ non-pigmented ascospores showed apparent recombination between the stu markers and the mating type factors (Table I). The apparent recombinant spores with stunted growths, no. 23 of cross no. 2, and nos. I, 3, 4, I6 and 17 of cross no. 3 , could not be classified as true recombinants without further study, since the stu markers could not be identified through complementation, and a I: I segregation could not be determined from the aborted $f_{2}$ asci and ascospores. Proof of their recombinant nature is provided in the crosses discussed below.

(I) Cross no. 3, ascospore no. 3 (Table I). This spore, an apparent recombinant, produced a culture which had a stunted growth and carried the $A$ mating type allele. The backcross reactions with both Sordaria heterothallis and $S$. thermophila were interspecific. The $S$. thermophila backcross produced aborted asci with no germinable ascospores. In the $S$. heterothallis backcross, a few aborted asci with I to 2 mature spores were produced, and one mature eight-spored ascus was recovered. The eight black spores which germinated were wild type in growth and $a$ in mating type. Three non-pigmented germinable spores - two of stunted and one of wild type growthwere recovered and analysed. The stunted strains were determined to be $s t u_{5} a$ through complementation tests. The recovery of the $s t u_{5}$ marker in the backcross progeny proves that the original non-pigmented ascospore must have been recombinant, with the $s t u_{5}$ marker derived from $S$. heterothallis and the $A$ mating type locus from $S$. thermophila. This ascospore could only have resulted from an interspecific nuclear fusion. 
(2) Cross no. 3, ascospore no. I4 (Table I). This spore produced a mycelium with stunted growth, which was $a$ in mating type and gave a negative complementation test. The backcross with Sordaria heterothallis produced aborted asci with I to 2 mature ascospores; several non-pigmented germinable ascospores were isolated. Of these, two were wild type, one sub-wild type and four stunted in phenotype. One of these stunted strains was determined to be $s t u_{1} s t u_{4} a$, through complementation tests. Thus the $\mathrm{f}_{1}$ non-pigmented ascospore which was stunted in phenotype must have carried the $s t u$ markers. That these $f_{2}$ non-pigmented ascospores could take part in complementation reactions with $s t u$ strains of $S$. heterothallis reflects the fact that they contained a larger part of the $S$. heterothallis genome than the $f_{1}$ ascospores. That they were non-pigmented reflects the fact that they were the products of mixed genomes. From this result it may be concluded that ascospores no. I, 4, I6 and 17 from cross no. 3, which were phenotypically stunted, carried the stu markers and were recombinant. Ascospore no. I I was also shown to be recombinant for the $s t u_{5}$ marker and mating type factor, as is described in the section in which strains phenotypically resembling $S$. heterothallis are obtained through backcrossing.

Ascospore no. 2, cross no. I (Table I), and ascospores 21, 22, 24 and 25 of cross no. 2 were also interspecific recombinants. The initial backcross reactions of spore no. 2, cross no. I with Sordaria heterothallis and $S$. thermophila were interspecific. This strain was however repeatedly subcultured over 3 months while other experiments were in progress. On further testing, the backcross was found to produce an interspecific reaction with $S$. heterothallis but an intraspecific reaction with $S$. thermophila. This $\mathrm{f}_{1}$ segregant, $a \mathrm{MT}$, was clearly an interspecific recombinant.

These results demonstrated that the non-pigmented germinable ascospores contained a mixture of the genomes of Sordaria heterothallis and $S$. thermophila. Such mixtures appeared to impair some of the physiological functions of the cells; spore pigmentation is prevented, and stu strains failed to show complementation either among themselves or when mixed with stu strains of $S$. heterothallis. Proof that these strains were the products of mixed genomes was also shown by the recovery of both of the original species from them as is demonstrated below.

\section{Formation of strains phenotypically resembling $S$. thermophila from a non-pigmented ascospore}

Strains phenotypically resembling Sordaria thermophila were obtained from $\mathrm{f}_{\mathbf{1}}$ nonpigmented ascospore cultures by successive backcrossing to the $S$. thermophila wild type strain. One such series of backcrosses (Fig. I) was started with the culture from ascospore no. I of cross no. 3 (Table I). The mycelium from this ascospore had a stunted growth and was $A$ in mating type. Only non-pigmented ascospores were recovered from the interspecific backcross with $S$. heterothallis but none could be induced to germinate. The backcross with $S$. thermophila produced two types of perithecia. (I) Several perithecia contained asci with I to 8 black spores (Pl. I, fig. 3). Six asci with eight black spores were isolated; the ascospores germinated on ammonium only after the $2-\mathrm{hr}$ heat treatment at $50^{\circ}$. All germinating ascospores were wild type in growth, although one parent appeared to carry the stu marker. This and the fact that this culture gave rise to two types of perithecia in this cross suggests that strain I of cross no. 3 was a heterocaryotic culture. Figure I shows the results of the analysis of ascus no. 4 of the $f_{2}$ backcross. Both mating type factors were recovered. 


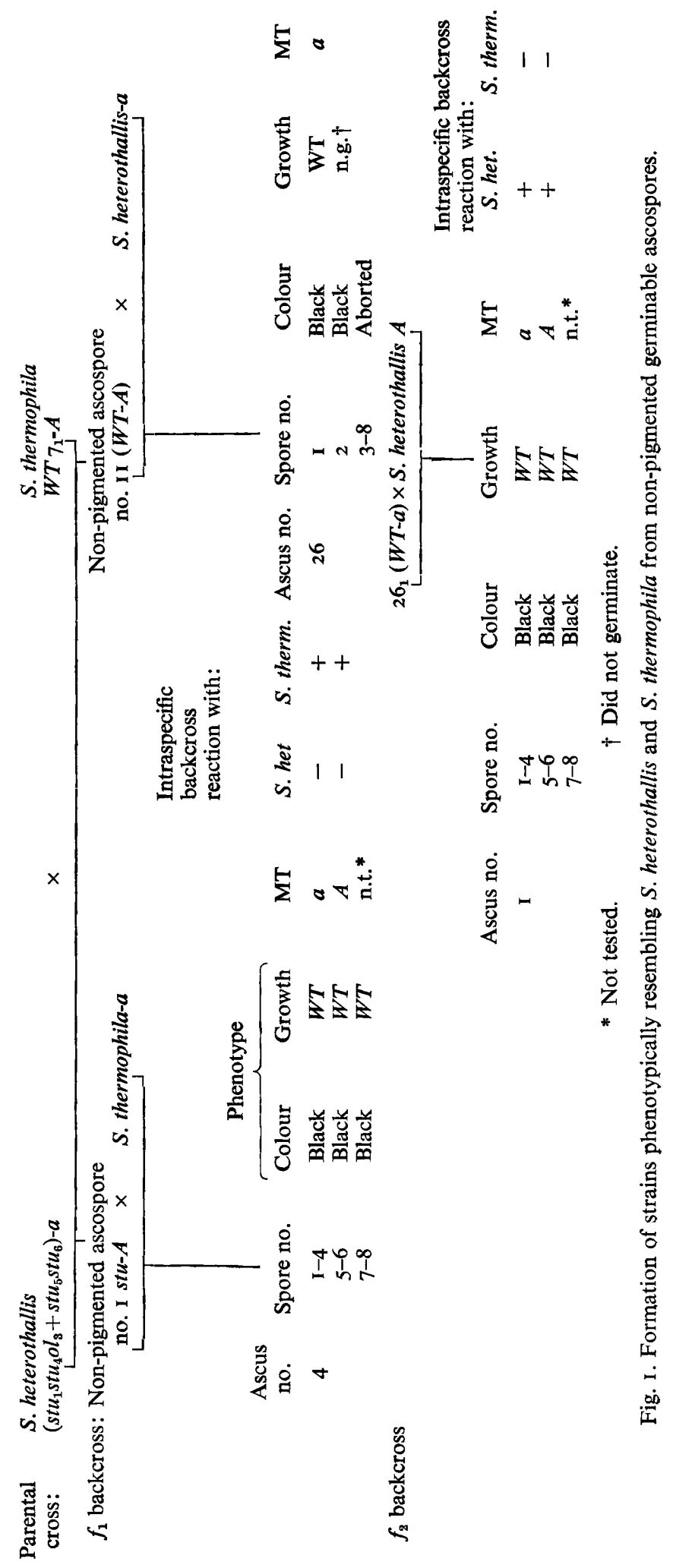


Mycelia from each of three pairs of ascospores of this ascus were backcrossed to the wild type strains of $S$. heterothallis and $S$. thermophila respectively. Normal segregation of the mating type alleles was observed in all crosses with $S$. thermophila, with asci and ascospores showing the typical shape and size of this species (P1. I, fig. 5). Strains with the $S$. thermophila phenotype were thus isolated among the $f_{3}$ progeny. (2) The majority of $f_{1}$ perithecia in this backcross contained asci which showed an interspecific reaction, with some asci containing I to 2 black ascospores (Pl. I, fig. 4). One mature eight-spored ascus which was recovered from these perithecia was homozygous for the wild type growth and $a$ mating type of $S$. thermophila. This confirms the results described previously (Lewis, 1969) that the formation of the mature eight-spored ascus was exclusively an intraspecific event.

\section{Formation of strains phenotypically resembling $S$. heterothallis from a non-pigmented ascospore}

Strains phenotypically resembling Sordaria heterothallis were obtained from recombinant $f_{1}$ non-pigmented germinable ascospores through successive backcrosses to the $S$. heterothallis wild type. Figure I shows a series of backcrosses which were started with ascospore culture no. I I of cross no. 3 (Table $r$ ). This spore produced a wild type mycelium of $A$ mating type. Backcrosses to $S$. heterothallis and $S$. thermophila were both interspecific, but perithecia from the former produced numerous aborted asci with I to 4 black ascospores. Cultures from eight of the 16 isolated black spores were $s t u_{5} a$ in genotype. Thus the original $f_{1}$ ascospore was interspecifically recombinant. One of the wild type $\mathrm{f}_{2}$ ascospores of $a$ mating type was backcrossed to $S$. heterothallis $A$. The $\mathrm{f}_{3}$ asci contained mostly 4 to 8 black spores (Pl. I, fig. 6). Six asci with eight black spores were dissected, and all ascospores showed wild type growth. Figure I shows the analysis of ascus no. I. A mycelium from each of three spore pairs was backcrossed to both $S$. heterothallis and $S$. thermophila; all produced intraspecific reactions with $S$. heterothallis (Pl. I, fig. 7 ) and interspecific reactions with $S$. thermophila. Strains with the $S$. heterothallis phenotype were thus isolated among the $f_{4}$ progeny. The replacement of parts of the genomes of the non-pigmented germinable ascospores with parts of the genomes of $S$. heterothallis or $S$. thermophila, the demonstration of interspecific recombination and the fact that these spores react inter-specifically with both species, is evidence that these non-pigmented spores are inter-specific recombinants.

\section{DISCUSSION}

The non-pigmented germinable ascospore is a cell containing parts of the genomes of the two species Sordaria heterothallis and $S$. thermophila. The mixture of chromosomes appears to impair the growth rate in some strains and inhibits spore pigmentation in all cases. It is probable that the synthesis of the spore pigment is controlled by several loci on a number of chromosomes, and that the necessary complementation between the products of these loci which is needed for pigment synthesis cannot or can only partially take place, as in the production of beige coloured germinable ascospores, when the chromosomes are from different species. This may reflect the evolutionary differences between the chromosomes of these two species. A hypothesis of this nature can be formulated on the basis of the results obtained from attempts to force heterocaryons between strains carrying the $s t u$ markers. If one or both of these strains is 
derived from a non-pigmented germinable ascospore no complementation takes place, although the stu markers were originally derived from the same species. It cannot be assumed that the differences in cytoplasms may be responsible for this result; for example, the inability of $S$. heterothallis proteins to be formed on $S$. thermophila polysomes. Lewis (1969) has shown that the genome of $S$. heterothallis can express itself normally in the cytoplasm of $S$. thermophila and vice versa. Another explanation for these irregular phenotypic expressions may be that the nuclei in these ascospores are aneuploid. This appears to be the explanation for the ascospore culture no. 2 of cross no. I, which may have lost its extra chromosomes of $S$. heterothallis during successive subculturing. In this case, however, the $a$ mating type allele from $S$. heterothallis appears to have been incorporated into the mating type chromosome of $S$. thermophila resulting in a recombinant genome. In the genus Neurospora Dodge (193I) was able to introduce certain factors from $N$. sitophila into $N$. crassa and show their normal segregation in eight-spored asci from the $\mathrm{f}_{1} \times N$. crassa backcross. More detailed work along these lines in $N$. crassa has been done by Fincham (I95I), and Scott-Emuakpor (I965), who have compared the recombination frequencies of markers first in one species and then after transfer into the genome of the other. One result of the presence of aneuploid nuclei would be the eventual formation of heterocaryotic strains (Pittenger, 1954) as in ascospore culture no. II of cross no. 3. This phenotypically wild type culture was shown to contain nuclei containing the $s t u_{5}$ marker. On the other hand the phenotypically stunted culture of ascospore no. I cross no. 3 when crossed with the wild type strain of $S$. thermophila gave rise to asci with eight spores which formed cultures with wild type growth. This suggested that only nuclei without the $S$. heterothallis stu marker took part in the formation of these asci.

The recovery of Sordaria heterothallis and S. thermophila from recombinant ascospores by backcrossing is similar in some respects to the work done with Neurospora by Dodge (1928, 1931, 1936).

The hybrid asci obtained from crosses between Neurospora stiophila and N. tetrasperma (Dodge, 1928) showed less abortion than did those in the hybridization of our Sordaria species. There was better delimitation of ascospores, and a large number of mature ascospores were obtained, although the number of perithecia produced seems to have been very small. Dodge was able to recover $N$. tetrasperma after two backcrosses, as has been the case with Sordaria thermophila in the present study. Dodge recovered the $N$. sitophila genome by intercrossing certain $f_{1}$ isolates. When $f_{1}$ from interspecific crosses in Sordaria were crossed among themselves the asci contained more rather than fewer aborted ascospores.

Hybridization studies in Sordaria suggest that these two species are less closely related than the two species, Neurospora sitophila and $N$. crassa. A large number of $\mathrm{f}_{1}$ ascospores with mixed genomes which were recovered from the Neurospora hybridization appeared morphologically and physiologically normal, and markers introduced from both species were expressed normally (Dodge, 193I). Very similar results were obtained in crosses involving $N$. sitophila and N. tetrasperma (Dodge, I93 I, 1936). The fact that strains from the $f_{1}$ non-pigmented ascospores reacted interspecifically with both species of Sordaria, although they were composed of parts of the genomes of both species, as has been shown through the results of genetic analysis, suggests that there must be very little affinity between the two species. 
The author wishes to express his appreciation to Professor Lindsay S. Olive for his guidance and support, and for his many helpful suggestions during the course of these studies, and to Dr W. G. Fields for his advice and suggestions. The willingness of Dr Fields to supply the cultures used in this study is gratefully acknowledged.

\section{REFERENCES}

Dodge, B. O. (1928). Production of fertile hybrids in the ascomycete Neurospora. J. agric. Res. 36, I. DoDGE, B. O. (I93I). Inheritance of the albinistic non-conidial characters in interspecific hybrids in Neurospora. Mycologia 23, I.

DodGe, B. O. (1936). Interspecific hybrids involving factors for ascus abortion in Neurospora. $A m$. J. Bot. 23, 555 .

FIELDS, W. G. (1963). Genetic investigation in the Sordaria heterothallis complex. Ph.D. Thesis. Wayne State University, Detroit, Michigan, U.S.A.

Fields, W. G. (I968). A new species of Sordaria. Mycologia 60, i 117.

Fields, W. G. \& MANiotis, J. (1963). Some cultural and genetic aspects of a new heterothallic Sordaria. Am. J. Bot. 50, 80 .

FINCHAM, J. R. S. (195I). A comparative genetic study of the mating type chromosomes of two species of Neurospora. J. Genet. 50, $22 \mathrm{I}$.

Lewis, L. A. (1968a). Genetics of an interspecific cross in the genus Sordaria. Genetics, Princeton 6o, 197 (Abstr.).

LewIS, L. A. (I968b). Genetics of an interspecific cross in the genus Sordaria. Ph.D. Thesis. Columbia University, New York, U.S.A.

LEWIS, L. A. (1969). Genetics of an interspecific cross in the genus Sordaria I. Analysis of 8-spored asci homozygous for the mating-type factor. Genetics, Princeton (in the Press).

PItTenger, T. H. (1954). The general incidence of pseudo-wild types in Neurospora crassa. Genetics, Princeton 39, 326.

SCOTT-EMUAKPOR, M. B. (1965). Genetic recombination in Neurospora crassa and N. sitophila. Genet. Res. 6, 216.

\section{EXPLANATION OF PLATE}

\section{Plate I}

Fig. I. Portion of Fig. 2 enlarged to show non-pigmented ascospore germinating in an ascus plated in corn meal agar. $\times 2500$.

Fig. 2. Interspecific cluster from Sordaria heterothallis $\times S$. thermophila cross, showing asci with aborted ascospores in all stages of development. Note ascus with germinating ascospore. $\times 550$.

Fig. 3 to 4 . Magnifications, $\times 350$.

Fig. 3. Clusters of asci with I to 8 mature ascospores from the following cross: Mycelium from non-pigmented $\mathrm{f}_{1}$ ascospore no. I of cross no. 3 (Table I) $A \times S$. thermophila $a$.

Fig. 4. Cross similar to that in Fig. 3. Asci from perithecia which produced interspecific clusters.

Fig. 5 to 7 . Magnifications, $\times 350$.

Fig. 5. $\mathrm{f}_{3} S$. thermophila ascospores from the $\mathrm{f}_{2}$ back-cross of mycelium derived from spore of cluster shown in Fig. 3, to $S$. thermophila wild type.

Fig. 6. $\mathrm{f}_{3}$ asci mostly with 6 to 8 mature ascospores from the following cross: $\mathrm{f}_{2}$ ascospore progeny $a$ (Fig. 3 ascospore $26_{1}$ ) $\times S$. heterothallis $A$. Most of these $\mathrm{f}_{3}$ ascospores show a resemblance to $S$. heterothallis wild type ascospores.

Fig. $7 . \mathrm{f}_{4} S$. heterothallis ascospores obtained by crossing progeny from clusters like those in Fig. 6 with $S$. heterothallis wild type. 

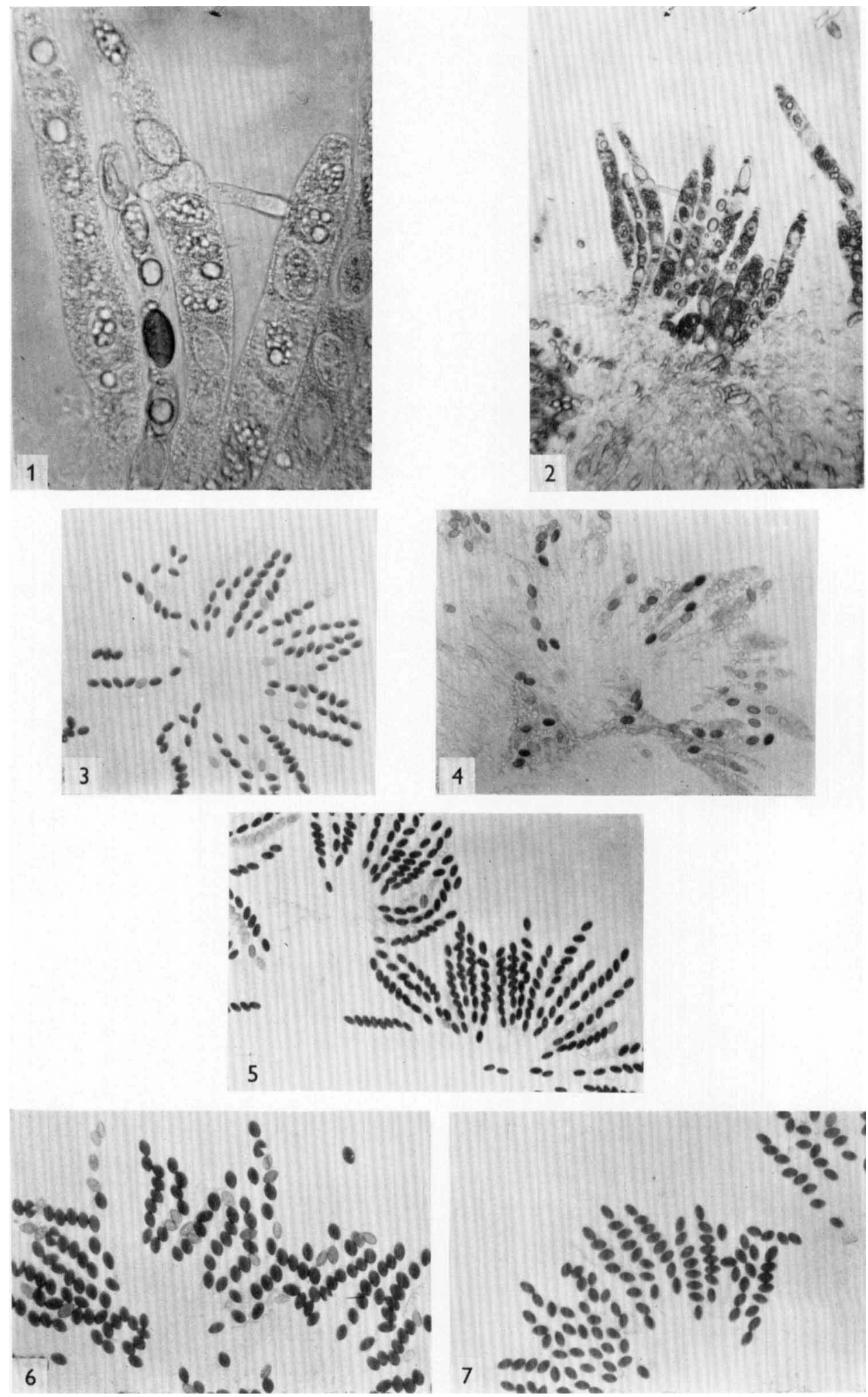